\title{
Application of uniform asymptotics to the connection formulas of the fifth Painlevé equation
}

\author{
Zhao-Yun Zeng and Yu-Qiu Zhao* \\ Department of Mathematics, Sun Yat-sen University, GuangZhou 510275, China
}

\begin{abstract}
We apply the uniform asymptotics method proposed by Bassom, Clarkson, Law and McLeod [4] to a special Painlevé V equation, and we provide a simpler and more rigorous proof of the connection formulas for a special solution of the equation, which have been established earlier by McCoy and Tang via the isomonodromy and WKB methods.
\end{abstract}

2010 Mathematics Subject Classification. 33E17, 33C10, 34E05.

Keywords and phrases: Connection formula; uniform asymptotics; the fifth Painlevé transcendent; parabolic cylinder function; Bessel function.

\section{Introduction}

We apply and extend the method of uniform asymptotics proposed by Bassom, Clarkson, Law and McLeod in [4] to a special case of the fifth Painlevé equation (PV)

$$
\frac{d^{2} y}{d x^{2}}=\left(\frac{1}{2 y}+\frac{1}{y-1}\right)\left(\frac{d y}{d x}\right)^{2}-\frac{1}{x} \frac{d y}{d x}+(1-2 \Theta) \frac{y}{x}-\frac{y(y+1)}{2(y-1)}, \quad \Theta \in \mathbb{R} \backslash \mathbb{Z},
$$

which appears in the time-dependent correlation functions of the transverse Ising chain at the critical value of the magnetic field [14, 15]. Our main focus will be on the connection formulas of the equation.

The equation (1.1) is reducible to a special case of the third Painlevé equation (PIII). Indeed, if we put $y(x)=\left(\frac{w(t)-1}{w(t)+1}\right)^{2}$ with $x=4 t$, then $w(t)$ satisfies Painlevé III, namely

$$
\frac{d^{2} w}{d t^{2}}=\frac{1}{w}\left(\frac{d w}{d t}\right)^{2}-\frac{1}{t} \frac{d w}{d t}+\frac{1-2 \Theta}{t}\left(w^{2}-1\right)+w^{3}-\frac{1}{w}
$$

\footnotetext{
*Corresponding author (Yu-Qiu Zhao). E-mail address: stszyq@mail.sysu.edu.cn
} 
which arose in Ising model studies [16]. McCoy, Tracy and Wu [16] derived $\infty \leftrightarrow 0$ connection formulas for a two-parameter class of bounded solutions $w(t ; \Theta, \rho)$ of the one-parameter family of Panlevé III equations (1.2).

The special Painlevé V equation (1.1), or the equivalent Painlevé III equation (1.2), plays a crucial role in problems related to random matrices and random processes, orthogonal polynomials, string theory, and in exactly solvable statistical mechanics and quantum field models. For example, for the special case $\Theta \rightarrow 0$, if we set $w(t)=-e^{i \phi(t)}$ in (1.2), then $\phi(t)$ satisfies the following equation

$$
\left(t \phi^{\prime}(t)\right)^{\prime}-2 t \sin 2 \phi(t)+2 \sin \phi(t)=0,
$$

which appeared in the problem of classification for rotation surfaces with harmonic inverse mean curvature [3]. This equation has been numerically investigated by Bobenko et al. [3], and also studied by Andreev and Kitaev [1] based on the results obtained in [2] by using isomonodromy deformation and the WKB method.

The problem concerning one particle density matrix of impenetrable bosons at zero temperature (see Creamer et al. [5], and Vaidya and Tracy [25]) has been reduced in [5] to a study of the equation

$$
\varphi^{\prime \prime}(z)=\left(\left(\varphi^{\prime}(z)\right)^{2}-1\right) \cot \varphi(z)+\frac{1}{z}\left(1-\varphi^{\prime}(z)\right) .
$$

Creamer et al. [5] have studied the solution of the equation numerically. An analytical study of (1.4) has carried out by Suleimanov [23].

It is worth pointing out that the equation (1.4) is equivalent to equation (1.3) via the transformation of the dependent variables $e^{i \varphi(z)}=i \tan \frac{\phi(t)}{2}$ and the independent variables $z=2 i t$.

In another special case when $\Theta=\frac{1}{2}$, we may take the transformation $w(t)=e^{i \psi(t)}$, and then the Painlevé III equation (1.2) becomes

$$
\psi^{\prime \prime}(t)+\frac{1}{t} \psi^{\prime}(t)=2 \sin 2 \psi(t) .
$$

For a numerical study of the last equation, see Lamb [11], with a connection to the $\pi$ pulse of the sine-Gordon equation. Flaschka and Newell [8] have also considered equation (1.5) by using the methods of monodromy preserving deformation and singular integral equations, and obtained representation of the one-parameter family of solutions to (1.5) that are holomorphic at the origin.

The same differential equation (1.5), adapting the new variable $s=4 t^{2}$, is a special case of the following equation

$$
\psi^{\prime \prime}+\frac{1}{s} \psi^{\prime}=\frac{1}{8 s} \sin (2 \psi)-\frac{\tilde{\alpha}^{2}}{4 s^{2}} \frac{\cos \psi}{\sin ^{3} \psi}
$$

with $\tilde{\alpha}=0$, which is closely related to the asymptotics of Bessel kernel limit of the Fredholm determinant describing the statistics of the level spacing of the eigenvalues of Hermitian matrices of large order in a single interval; see Tracy and Widom [24]. 
Now we see that the Painlevé V (1.1) is related to various nonlinear equations with statistic physical backgrounds. Yet the main objective of the present paper, is to calculate the asymptotics of a special solution as as $x \rightarrow 0$ and $x \rightarrow+\infty$, and to justify connection formulas between parameters involved in the asymptotic approximations. It is known that there exist solutions to (1.1), regular on the positive real line, with behavior at the origin

$$
y(x)=\frac{i \rho+\Theta}{i \rho-\Theta}\left[1+(1-2 \Theta) x+O\left(x^{2}\right)\right] \quad \text { as } x \rightarrow 0
$$

cf. [15, 17], where the parameter $\rho$ is not on the imaginary axis, and such that $|\operatorname{Im} \rho| \geqslant \Theta$.

McCoy and Tang [17, 18] derived $+\infty \leftrightarrow 0$ and $\pm i \infty \leftrightarrow 0$ connection formulas for two-parameter solutions of the one-parameter family of Painlevé V equations (1.1), respectively. In this paper, we focus on the $+\infty \leftrightarrow 0$ connection problem. It is known from [15, 17] that the solution of (1.1) satisfying boundary condition (1.6) possesses the following asymptotic expansion

$$
y(x)=-1+4 x^{-\frac{1}{2}} F_{1}(s)+4 x^{-1}\left[2 \Theta-1-2 F_{1}^{2}(s)\right]+O\left(x^{-\frac{3}{2}}\right) \text { as } x \rightarrow+\infty,
$$

where

$$
F_{1}(s)=a e^{2 i s}+b e^{-2 i s}
$$

with $s=\frac{x}{4}-a b \ln \frac{x}{4}$. Here $a$ and $b$ are independent of $x$, and $|\operatorname{Im}\{a b\}|<\frac{1}{4}$. The following results state that the parameters $a$ and $b$ in (1.8) are explicit functions in the parameter $\rho$ in (1.6):

\section{Theorem 1.}

$$
\begin{aligned}
& a(\rho)=\frac{1}{2 \sqrt{\pi}} \Gamma(1+2 i c(\rho)) e^{\frac{i \pi}{4}} e^{-6 i c(\rho) \ln 2} e^{\pi c(\rho)}\left[\cos \pi \Theta-\frac{\rho}{\Theta} \sin \pi \Theta\right], \\
& b(\rho)=\frac{1}{2 \sqrt{\pi}} \Gamma(1-2 i c(\rho)) e^{-\frac{i \pi}{4}} e^{6 i c(\rho) \ln 2} e^{\pi c(\rho)}\left[\cos \pi \Theta+\frac{\rho}{\Theta} \sin \pi \Theta\right],
\end{aligned}
$$

where

$$
c(\rho)=-\frac{1}{4 \pi} \ln \left[\left(1+\frac{\rho^{2}}{\Theta^{2}}\right) \sin ^{2} \pi \Theta\right] .
$$

Remark 1. From (1.9) and (1.10) it is readily observed that

$$
a(\rho) b(\rho)=c(\rho) .
$$

Here use has been made of the fact that $\Gamma(1+2 i c) \Gamma(1-2 i c)=\frac{4 \pi c}{e^{2 \pi c}-e^{-2 \pi c}}$. From (1.11) and (1.12), we see that $|\operatorname{Im}\{a b\}|<\frac{1}{4}$ if and only if $\rho$ is not on the parts of imaginary axis with $|\operatorname{Im} \rho| \geqslant \Theta$. 
The above connection formulas have been established by McCoy and Tang in [17] by using the method of isomonodromy deformation and the matching of WKB solutions. To explain their approach, we briefly outline the relation of Painlevé $\mathrm{V}$ with the theory of monodromy preserving deformations of linear ordinary differential equations with rational coefficients. The reader is referred to [7, 10] for more details.

The Lax pair of the fifth Painlevé equation, with parameters $\Theta_{0}=\Theta_{1}=\Theta$ and $\Theta_{\infty}=0$, is a system of linear differential equations for the matrix function $\Psi(\lambda, x)$,

$$
\frac{\partial \Psi}{\partial \lambda}=\left(\begin{array}{cc}
\frac{x}{2}+\frac{v+\frac{\Theta}{2}}{\lambda}-\frac{v+\frac{\Theta}{2}}{\lambda-1} & -\frac{u(v+\Theta)}{\lambda}+\frac{y u v}{\lambda-1} \\
\frac{v}{u \lambda}-\frac{v+\Theta}{u y(\lambda-1)} & -\frac{x}{2}-\frac{v+\frac{\Theta}{2}}{\lambda}+\frac{v+\frac{\Theta}{2}}{\lambda-1}
\end{array}\right) \Psi
$$

and

$$
\frac{\partial \Psi}{\partial x}=\left(\begin{array}{cc}
\frac{\lambda}{2} & -\frac{u}{x}(v+\Theta-y v) \\
\frac{1}{x u}\left(v-\frac{v+\Theta}{y}\right) & -\frac{\lambda}{2}
\end{array}\right) \Psi,
$$

where $y(x), v(x)$ and $u(x)$ satisfy the following isomonodromy deformation system:

$$
\begin{aligned}
& x \frac{\mathrm{d} y}{\mathrm{~d} x}=x y-2 v(y-1)^{2}+2 \Theta(y-1), \\
& x \frac{\mathrm{d} v}{\mathrm{~d} x}=y v^{2}-\frac{1}{y}(v+\Theta)^{2}, \\
& x \frac{\mathrm{d}}{\mathrm{d} x} \ln u=-2 v-\Theta+y v+\frac{1}{y}(v+\Theta) .
\end{aligned}
$$

Furthermore, $y(x)$ solves the fifth Painlevé equation (1.1). In this sense, the Painlevé V equation is equivalent to the compatibility condition $\Psi_{\lambda x}=\Psi_{x \lambda}$.

The canonical solutions $\Psi_{k}=\Psi_{k}(\lambda)$ of (1.13), $k=1,2$, are determined by their asymptotic approximations. In a neighborhood of the irregular singular point $\lambda=\infty$, they have the following asymptotic expansion:

$$
\Psi_{k}^{(\infty)}(\lambda)=\left(I+O\left(\frac{1}{\lambda}\right)\right) \exp \left(\frac{\lambda x}{2} \sigma_{3}\right),|\lambda| \rightarrow \infty, x \in \mathbb{R}^{+}, k=1,2,
$$

respectively in the Stokes sectors $\Omega_{1}$ and $\Omega_{2}$ defined as

$$
\Omega_{k}=\left\{\lambda:-\frac{\pi}{2}+\pi(k-2)<\arg \lambda<\frac{3 \pi}{2}+\pi(k-2),|\lambda|>R\right\}, \quad k=1,2,
$$

with arbitrary finite positive constant $R$.

These functions are related by certain Stokes matrices $S_{1}$ and $S_{2}$, namely,

$$
\Psi_{2}^{(\infty)}(\lambda)=\Psi_{1}^{(\infty)}(\lambda) S_{1}, \quad \Psi_{1}^{(\infty)}(\lambda)=\Psi_{2}^{(\infty)}\left(e^{2 \pi i} \lambda\right) S_{2},
$$

where

$$
S_{1}=\left(\begin{array}{cc}
1 & 0 \\
s_{1} & 1
\end{array}\right), \quad S_{2}=\left(\begin{array}{cc}
1 & s_{2} \\
0 & 1
\end{array}\right)
$$


and the constants $s_{1}$ and $s_{2}$ are called the Stokes multipliers.

The canonical solutions at $\lambda=0$ and $\lambda=1$ are $2 \times 2$ unimodular matrices $\Psi^{(0)}(\lambda, x)$ and $\Psi^{(1)}(\lambda, x)$, and can be defined as

$$
\Psi_{1}^{(\infty)}(\lambda, x)=\Psi^{(0)}(\lambda, x) E_{0}, \quad \Psi_{1}^{(\infty)}(\lambda, x)=\Psi^{(1)}(\lambda, x) E_{1}
$$

where $E_{0}$ and $E_{1}$ are unimodular constant matrices. Furthermore, the behavior at the singularities are

$$
\begin{array}{ll}
\Psi^{(0)}(\lambda, x)=\tilde{\Psi}^{(0)}(\lambda, x) \lambda^{\frac{1}{2} \Theta \sigma_{3}} & \text { as } \lambda \rightarrow 0, \\
\Psi^{(1)}(\lambda, x)=\tilde{\Psi}^{(1)}(\lambda, x)(\lambda-1)^{\frac{1}{2} \Theta \sigma_{3}} & \text { as } \lambda \rightarrow 1,
\end{array}
$$

with $\tilde{\Psi}^{(0)}(\lambda, x)$ and $\tilde{\Psi}^{(1)}(\lambda, x)$ being holomorphic at $\lambda=0$ and $\lambda=1$, respectively.

The monodromy matrices $E_{k}$ at the regular singularities $\lambda=k(k=0,1)$ fulfil the following cyclic relation:

$$
E_{0}^{-1} e^{-\pi i \Theta \sigma_{3}} E_{0}=S_{1} S_{2} E_{1}^{-1} e^{\pi i \Theta \sigma_{3}} E_{1}
$$

which specifies $E_{0}$ and $E_{1}$ up to left-multiplicative diagonal matrices $\operatorname{diag}\left(d_{0}, d_{0}^{-1}\right)$ and $\operatorname{diag}\left(d_{1}, d_{1}^{-1}\right)$, respectively. The connection matrices $E_{0}$ and $E_{1}$ are independent of $x$ : The isomonodromic condition $\frac{d E_{k}}{d x}=0$ holds here owing to the fact that all of $\Psi_{1}^{(\infty)}(\lambda, x)$, $\Psi^{(0)}(\lambda, x)$ and $\Psi^{(1)}(\lambda, x)$ are solutions of (1.14). We note that none of the entries in matrices $E_{0}$ and $E_{1}$ vanishes. In fact, for example, if $\left(E_{0}\right)_{21}=0$, then from [2] we see that all the Stokes multipliers $s_{k}=0, k=1,2$. From the results in [10] for the connection matrices and in view of (1.23), we obtain $\rho=i \Theta$, which contradicts the restriction on $\rho$.

Appealing to the isomonodromic deformation techniques developed in [9], we may use $E_{0}$ and $E_{1}$ to express the parameters $a$ and $b$ in (1.7) as functions of the initial parameter $\rho$ in (1.6). More exactly, the quantities to be used are

$$
I_{0}=\frac{\left(E_{0}\right)_{11}\left(E_{0}\right)_{22}}{\left(E_{0}\right)_{12}\left(E_{0}\right)_{21}}
$$

and

$$
I_{1}=\frac{\left(E_{1}\right)_{11}\left(E_{1}\right)_{22}}{\left(E_{1}\right)_{12}\left(E_{1}\right)_{21}} .
$$

As mentioned earlier, the connection problem (1.9)-(1.10) was first established by McCoy and Tang [17]. They considered the asymptotic behavior of the solution $\Psi(\lambda, x)$ to the first order system (1.13) as $x \rightarrow \infty$ for $\lambda$ being kept away from the turning point $\frac{1}{2}$ and the singular points 0,1 . Then, they matched these WKB solutions with the asymptotic approximation at the turning point involving parabolic cylinder functions, and the behavior at the singularities $\lambda=0,1$ involving the Bessel functions. Eventually they were capable of calculating the two invariants (1.24) and (1.25) as $x \rightarrow+\infty$. The 
invariants $I_{0}$ and $I_{1}$ for small $x$ can be obtained from the connection matrices $E_{0}$ and $E_{1}$, which have been done by Jimbo [10]; see also [17, 19], such that

$$
\begin{aligned}
& I_{0}=\frac{i \rho+\Theta}{i \rho-\Theta}, \text { and } \\
& I_{1}=\frac{i \rho-\Theta}{i \rho+\Theta} .
\end{aligned}
$$

The facts that $I_{0}$ and $I_{1}$ are independent of $x$ will lead to equalities involving $a, b$ and the initial parameters. The formulas in Theorem 1 would then follow. The procedure, however, is complicated, and is difficult to make rigorous; see a comment made in [4, p.245].

In this paper, we shall provide a hopefully simpler and more rigorous derivation of the formulas (1.9)-(1.10) by using the method of uniform asymptotics proposed by Bassom, Clarkson, Law and McLeod [4]. Along the same lines we may find the work of Olver [20] and Dunster [6] for coalescing turning points. Initially in [4], PII has been taken as an example to illustrate the method. While the difficulty in extending the techniques for PII to other transcendents is also acknowledged by the authors of [4, p.244]. Yet the method has been applied to the connection problems for PIII (Sine-Gordon) and PIV; cf Wong and Zhang [27, 28, and has also been used to find the asymptotic behavior at infinity of the solutions to PIV [22] and PV [12, 13].

We briefly outline the uniform asymptotics approach to derive the connection formulas (1.9)-(1.10). First, we will obtain the second-order differential equation (2.6) from the Lax pair (1.13). Then, we substitute the known large- $x$ asymptotic behaviors of $y(x)$ and $v(x)$ into the second-order equation and obtain an approximate equation (2.9). The equation (2.9) has only two coalescing turning points which coalesce with a regular point. Thus, uniform asymptotic solutions are to be constructed in terms of the parabolic cylinder functions, uniformly for $\lambda$ on the Stokes curves as $x \rightarrow \infty$; cf. Olver [20], and see Theorem 2 in Section 2 below.

Accordingly, we will calculate the connection matrix via (1.20) by using the asymptotics of the fundamental solutions on the Stokes curves; cf. Theorem 2, respectively as $|\lambda| \rightarrow \infty$ and $\lambda \rightarrow 0$ (or $\lambda \rightarrow 1$ ), for $x \rightarrow+\infty$. Consequently, we can use (1.24)-(1.25) to obtain $I_{0}$ and $I_{1}$ for large $x$; see (3.52)-(3.53) below. Finally, equating (1.26) and (3.52) gives $b=b(\rho)$, and equating (1.27) and (3.53) gives $a=a(\rho)$.

The difference between the method of uniform asymptotics and the WKB method is that the latter has to match different approximations in different regions while in the uniform asymptotics cases the complicated matching procedure is not needed. The derivation of the method of uniform asymptotics is rigorous and may lead to a simpler argument with minimal computational efforts. However, for the uniform asymptotics method, difficulties may arise in describing the geometry of the Stokes curves near the turning points in the finite plane, such as determining of the correspondence between $\pm \alpha$ and $\lambda_{1,2}$ in (2.15).

The rest of the paper is organized as follows. In Section 2 , we derive uniform ap- 
proximations to the solutions of the second-order differential equation obtained from the Lax pair (1.13) as $x \rightarrow+\infty$ by virtue of the parabolic cylinder functions on the Stokes curves. The last section is devoted to the evaluation of the two invariants $I_{0}$ and $I_{1}$ in (1.24)-(1.25) for large $x$. The proof of Theorem 1 is also provided in that section.

\section{Uniform asymptotics as $x \rightarrow+\infty$}

In the present section, we apply the uniform asymptotic method to deal with the large$x$ behavior of the second-order differential equation (2.9) obtained from the Lax pair (1.13).

First, we eliminate the function $u(x)$ in $(1.13)$ by taking the following gauge transformation:

$$
\hat{\Psi}=M_{1} u^{-\frac{1}{2} \sigma_{3}} \Psi
$$

As a result, we have

$$
\frac{d \hat{\Psi}}{d \lambda}=\left(\begin{array}{cc}
A & B \\
C & -A
\end{array}\right) \hat{\Psi}
$$

where

$$
\begin{aligned}
& M_{1}=\frac{1}{\sqrt{2}}\left(\begin{array}{cc}
1 & i \\
i & 1
\end{array}\right), \\
& A=i\left(\frac{v+\frac{\Theta}{2}}{\lambda}-\frac{y v+\frac{1}{y}(v+\Theta)}{2(\lambda-1)}\right), \\
& B=-i\left(\frac{x}{2}+\frac{v+\frac{\Theta}{2}}{\lambda}-\frac{v+\frac{\Theta}{2}}{\lambda-1}\right)+\frac{1}{2}\left(-\frac{\Theta}{\lambda}+\frac{1}{\lambda-1}\left(y v-\frac{v+\Theta}{y}\right)\right), \\
& C=i\left(\frac{x}{2}+\frac{v+\frac{\Theta}{2}}{\lambda}-\frac{v+\frac{\Theta}{2}}{\lambda-1}\right)+\frac{1}{2}\left(-\frac{\Theta}{\lambda}+\frac{1}{\lambda-1}\left(y v-\frac{v+\Theta}{y}\right)\right) .
\end{aligned}
$$

It is easily verified that the gauge transformation (2.1) does not change the monodromy matrix $E_{k}, k=0,1$.

Let $\left(\psi_{1}, \psi_{2}\right)^{T}$ be a vector solution of (2.2), and set

$$
\phi=B^{-\frac{1}{2}} \psi_{1}
$$

then $\phi(\lambda)$ solves the equation

$$
\frac{d^{2} \phi}{d \lambda^{2}}=\left[A^{2}+B C+A^{\prime}-A B^{-1} B^{\prime}+\frac{3}{4}\left(B^{-1} B^{\prime}\right)^{2}-\frac{1}{2} B^{-1} B^{\prime \prime}\right] \phi,
$$

where the derivatives are taken with respect to $\lambda$, for instance, $A^{\prime}=\frac{d A}{d \lambda}$. 
Combining (1.15) and (1.16) with (1.7), we have

$$
v(x)=-\frac{x}{8}-\frac{i}{4} x^{\frac{1}{2}} F_{2}(s)+\frac{1}{2}\left[F_{1}^{2}(s)-\Theta\right]+O\left(x^{-\frac{1}{2}}\right) \text { as } x \rightarrow+\infty,
$$

where $F_{1}(s)$ is defined in (1.8), $s=\frac{x}{4}-a b \ln \frac{x}{4}$, and

$$
F_{2}(s)=a e^{2 i s}-b e^{-2 i s} .
$$

For $x \rightarrow+\infty$, substituting (2.4) into (2.6), and in view of (1.7) and (2.7), a straightforward calculation yields the following second-order equation:

$$
\frac{d^{2} \phi}{d \lambda^{2}}=-x^{2}\left\{-\frac{(2 \lambda-1)^{2}}{16 \lambda(\lambda-1)}+\frac{Q_{1}(\lambda, x)}{x}-\frac{Q_{2}(\lambda, x)}{x^{3 / 2}}+O\left(x^{-2}\right)\right\} \phi:=-x^{2} Q(\lambda, x) \phi
$$

where

$$
\begin{aligned}
& Q_{1}(\lambda, x)=\frac{1}{4 \lambda(\lambda-1)}\left[4 a b+i-i\left(\lambda-\frac{1}{2}\right) \frac{H^{\prime}}{H}\right], \\
& Q_{2}(\lambda, x)=\frac{1}{2 \lambda(\lambda-1)}\left[F_{1}(s)+F_{2}(s)-\left(\lambda-\frac{1}{2}\right) F_{2}(s) \frac{H^{\prime}}{H}\right],
\end{aligned}
$$

with

$$
H(\lambda, x)=i x^{1 / 2}\left(\lambda-\frac{1}{2}\right)^{2}+\lambda F_{1}(s)-\frac{1}{2} F_{2}(s)+O\left(x^{-1 / 2}\right) \text { as } x \rightarrow \infty .
$$

Here $F_{1}(s)$ and $F_{2}(s)$ are given in (1.8) and (2.8), respectively.

For large $x$, equation (2.9) has two turning points

$$
\lambda_{j}=\frac{1}{2} \mp x^{-1 / 2} \sqrt{4 a b+i}(1+o(1)), \quad j=1,2,
$$

which coalesce with each other at $\frac{1}{2}$ as $x \rightarrow \infty$. The Stokes curves are defined as

$$
\operatorname{Re} \sqrt{\lambda(\lambda-1)}=0
$$

In line with the idea of uniform asymptotics in [4], we define a number $\alpha$ such that

$$
\frac{1}{2} \pi i \alpha^{2}=\int_{-\alpha}^{\alpha}\left(\tau^{2}-\alpha^{2}\right)^{1 / 2} d \tau=\int_{\lambda_{1}}^{\lambda_{2}} Q^{1 / 2}(\lambda, x) d \lambda
$$

and a new variable $\zeta$ by

$$
\int_{\alpha}^{\zeta}\left(\tau^{2}-\alpha^{2}\right)^{1 / 2} d \tau=\int_{\lambda_{2}}^{\lambda} Q^{1 / 2}(\eta, x) d \eta
$$

Here and in (2.15), the cut for the integrand on the left-hand side is the line segment joining $-\alpha$ and $\alpha$. The path of integration is taken along the upper edge of the cut. With $\alpha$ and $\zeta$ so chosen, the result in [4, Theorem 1] applies. Thus we have the following theorem. 
Theorem 2. Given a solution $\phi(\lambda, x)$ of (2.9), there exist constants $c_{1}$ and $c_{2}$ such that

$$
\left(\frac{\zeta^{2}-\alpha^{2}}{Q(\lambda, x)}\right)^{-\frac{1}{4}} \phi(\lambda, x)=\left\{\left[c_{1}+o(1)\right] D_{\nu}\left(e^{\pi i / 4} \sqrt{2 x} \zeta\right)+\left[c_{2}+o(1)\right] D_{-\nu-1}\left(e^{-\pi i / 4} \sqrt{2 x} \zeta\right)\right\}
$$

as $x \rightarrow+\infty$, uniformly for $\lambda$ on the Stokes curves defined in (2.14), where $D_{\nu}(z)$ and $D_{-\nu-1}(z)$ are parabolic cylinder functions; cf. [21, (12.2.5)], with

$$
\nu=-\frac{1}{2}+\frac{1}{2} i x \alpha^{2}
$$

\section{The monodromy data for $x \rightarrow+\infty$}

We proceed to calculate the two invariants $I_{0}$ and $I_{1}$ in $(1.24)-(1.25)$ as $x \rightarrow+\infty$. For our purpose, we need to clarify the relation between $\zeta$ and $\lambda$ in (2.16).

Lemma 1. For large $x$ and $\lambda$,

$$
\frac{1}{2} i x \zeta^{2}-\frac{1}{2} i x \alpha^{2} \ln \zeta=-\frac{x}{2} \lambda+\frac{i}{4} x+\frac{x}{4}+\pi a b-\frac{1}{4} \ln x+\frac{1}{2} \ln \left(b e^{-2 i s}\right)+o(1),
$$

where

$$
\alpha^{2}=\frac{4 a b+i}{x}+o\left(\frac{1}{x}\right) \quad \text { as } x \rightarrow \infty .
$$

Remark 2. Coupling (2.18) and (3.2) determines the approximate value

$$
\nu=2 i a b-1+o(1) \quad \text { as } x \rightarrow \infty
$$

for the order of the parabolic cylinder function $D_{\nu}\left(e^{\pi i / 4} \sqrt{2 x} \zeta\right)$ in (2.17).

Proof. A straightforward integration on the left-hand side of (2.16) yields

$$
\int_{\alpha}^{\zeta}\left(\tau^{2}-\alpha^{2}\right)^{1 / 2} d \tau=\frac{1}{2}\left\{\zeta\left(\zeta^{2}-\alpha^{2}\right)^{1 / 2}-\alpha^{2} \ln \left(\zeta+\left(\zeta^{2}-\alpha^{2}\right)^{1 / 2}\right)+\alpha^{2} \ln \alpha\right\} .
$$

Here, the cut for the integrand is again the line segment joining $-\alpha$ and $\alpha$, and again we take the integration path along the upper edge of the cut. In view of (3.4) and picking up the leading terms in (2.16), for large $\zeta$ we have

$$
\frac{1}{2} \zeta^{2}-\frac{1}{2} \alpha^{2} \ln (2 \zeta)-\frac{1}{4} \alpha^{2}+\frac{1}{2} \alpha^{2} \ln (\alpha)+O\left(\alpha^{4} \zeta^{-2}\right)=\int_{\lambda_{2}}^{\lambda} Q^{1 / 2}(\eta, x) d \eta .
$$

To calculate the right-hand side of (3.5), we split the integration interval, so that

$$
\int_{\lambda_{2}}^{\lambda} Q^{1 / 2}(\eta, x) d \eta=\left\{\int_{\lambda_{2}}^{\lambda^{*}}+\int_{\lambda^{*}}^{\lambda}\right\} Q^{1 / 2}(\eta, x) d \eta:=I_{1}+I_{2}
$$


where

$$
\lambda^{*}=\frac{1}{2}+T x^{-1 / 2},
$$

with $T$ being a large parameter to be specified more precisely later. When $\lambda$ approaches $1 / 2$, it follows from (2.10) and (2.11) that $Q_{1} \sim-(4 a b+i)$ and $Q_{2} \sim-2\left(F_{1}(s)+F_{2}(s)\right)=$ $-4 a e^{2 i s}$, where $s=\frac{x}{4}-a b \ln \frac{x}{4}, F_{1}$ and $F_{2}$ are given respectively in (1.8) and (2.8). Thus, in view of $|\operatorname{Im}\{a b\}|<\frac{1}{4}$, we have

$$
\frac{Q_{2}}{x^{1 / 2}}=o(1) \quad \text { as } x \rightarrow \infty \text {. }
$$

To approximate $I_{1}$, we make the change of variables

$$
\eta-\frac{1}{2}=t x^{-1 / 2}
$$

replace $Q_{1}$ by $-(4 a b+i)$, and ignore $Q_{2}$. Then for large $x$, we have

$$
\begin{aligned}
I_{1} & =\int_{\lambda_{2}}^{\lambda^{*}} Q^{\frac{1}{2}}(\eta, x) d \eta=\frac{1}{x} \int_{\sqrt{4 a b+i}}^{T} \sqrt{t^{2}-(4 a b+i)^{2}}(1+o(1)) d t \\
& =\frac{T^{2}}{2 x}-\frac{4 a b+i}{4 x}-\frac{4 a b+i}{2 x} \ln (2 T)+\frac{4 a b+i}{4 x} \ln (4 a b+i)+o\left(\frac{1}{x}\right) .
\end{aligned}
$$

Here, as before, the cut for the second integral is the line segment joining $-\sqrt{4 a b+i}$ and $\sqrt{4 a b+i}$, and the path of integration is taken along the upper edge of the cut. Taking $T=-\sqrt{4 a b+i}$ in $I_{1}$ gives (3.2).

When $\lambda$ is large, the three terms in the square brackets on the righthand side of (2.11) are of size $o\left(x^{1 / 2}\right)$ as $x \rightarrow \infty$. Comparing this with (2.10), one can ignore $Q_{2}$ in $I_{2}$; cf. (2.9) and (3.6). Accordingly we obtain

$$
\begin{aligned}
I_{2} & \approx \int_{\lambda^{*}}^{\lambda}\left[-\frac{(2 \eta-1)^{2}}{16 \eta(\eta-1)}+\frac{1}{x}\left(\frac{4 a b+i}{4 \eta(\eta-1)}-i \frac{2 \eta-1}{8 \eta(\eta-1)}\right) \frac{H^{\prime}}{H}\right]^{\frac{1}{2}} d \eta \\
& \approx i \int_{\lambda^{*}}^{\lambda} \frac{2 \eta-1}{4 \sqrt{\eta(\eta-1)}}\left[1-\frac{1}{x} \frac{8}{(2 \eta-1)^{2}}\left(\frac{4 a b+i}{4}-\frac{i(2 \eta-1)}{8} \frac{H^{\prime}}{H}\right)\right] d \eta \\
& \approx \frac{i}{2} \lambda-\frac{i}{4}+\frac{1}{4}-\frac{T^{2}}{2 x}-\frac{i \pi(4 a b+i)}{4 x}-\frac{4 a b+i}{4 x} \ln \frac{x}{T^{2}}+\frac{i}{2 x} \ln \frac{i x^{1 / 2}}{b e^{-2 i s}}
\end{aligned}
$$

with error term being $o\left(x^{-1}\right)+O\left(\lambda^{-1}\right)+O\left(T^{4} x^{-2}\right)$, where the derivative $H^{\prime}$ is taken with respect to $\eta$. Now setting $T<x^{1 / 4}$, substituting (3.9) and (3.10) into (3.5), and combining the latter with (3.2), we obtain Lemma 1.

Now we have the relation between $\zeta$ and $\lambda$ from (2.16) for large $\lambda$. We also need to establish the following relation for small $\lambda$ :

Lemma 2. When $\lambda \rightarrow 0$, for large $x$ such that $x \sqrt{\lambda} \rightarrow \infty$ and $x \zeta^{2} \rightarrow \infty$,

$$
\frac{1}{2} i x \zeta^{2}-\frac{1}{2} i x \alpha^{2} \ln \zeta=\frac{i}{2} x \sqrt{\lambda}+\frac{i}{4} x-\frac{1}{4} \ln x-\frac{\pi i}{4}+\frac{1}{2} \ln \left(b e^{-2 i s}\right)+o(1),
$$

where $\alpha^{2}$ given in (3.2). 
Proof. Now, we assume that $\lambda \rightarrow 0$ and $x$ is large such that $x \sqrt{\lambda} \rightarrow \infty$ and $x \zeta^{2} \rightarrow \infty$. Let

$$
\lambda_{*}=\frac{1}{2}-T_{*} x^{-1 / 2}
$$

where $T_{*}$ is a large positive constant to be specified later. We split the integral on the righthand side of (3.5) into three parts

$$
\int_{\lambda_{2}}^{\lambda} Q^{1 / 2}(\eta, x) d \eta=\left\{\int_{\lambda_{2}}^{\lambda_{1}}+\int_{\lambda_{1}}^{\lambda_{*}}+\int_{\lambda_{*}}^{\lambda}\right\} Q^{1 / 2}(\eta, x) d \eta:=I_{0}+I_{1}+I_{2} .
$$

On account of (2.15), we see that

$$
I_{0}=-\frac{\pi i}{2 x} \alpha^{2}+o\left(\frac{1}{x}\right)
$$

Similar to (3.9), we have

$$
\begin{aligned}
I_{1} & =\int_{\lambda_{2}}^{\lambda^{*}} Q^{\frac{1}{2}}(\eta, x) d \eta=\frac{1}{x} \int_{\sqrt{4 a b+i}}^{T} \sqrt{t^{2}-(4 a b+i)^{2}}(1+o(1)) d t \\
& =\frac{T_{*}^{2}}{2 x}-\frac{4 a b+i}{4 x}-\frac{4 a b+i}{2 x} \ln \left(2 T_{*}\right)+\frac{4 a b+i}{4 x} \ln (4 a b+i)+o\left(\frac{1}{x}\right) .
\end{aligned}
$$

When $\lambda \rightarrow 0$, for large $x$, from (2.11) we see that

$$
\int_{\lambda_{*}}^{\lambda} \frac{Q_{2} \sqrt{\lambda(\lambda-1)}}{x^{3 / 2}(2 \lambda-1)} d \lambda
$$

is of size $o\left(\sqrt{\lambda} x^{-1}\right)+o\left(x^{-1} \ln \left(\frac{x}{T_{*}^{2}}\right)\right)$. Then, using the binomial expansion, the integral $I_{2}$ in (3.13) is approximated as

$$
\begin{aligned}
I_{2} & \approx \int_{\lambda^{*}}^{\lambda}\left[-\frac{(2 \eta-1)^{2}}{16 \eta(\eta-1)}+\frac{1}{x}\left(\frac{4 a b+i}{4 \eta(\eta-1)}-i \frac{2 \eta-1}{8 \eta(\eta-1)}\right) \frac{H^{\prime}}{H}\right]^{\frac{1}{2}} d \eta \\
& \approx \frac{1}{2} \lambda+\frac{1}{4}-\frac{T_{*}^{2}}{2 x}+\frac{i \pi(4 a b+i)}{2 x}-\frac{4 a b+i}{4 x} \ln \frac{x}{T_{*}^{2}}+\frac{i}{2 x} \ln \frac{i x^{1 / 2}}{b e^{-2 i s}}
\end{aligned}
$$

with error term being $o\left(x^{-1}\right)+o\left(\sqrt{\lambda} x^{-1}\right)+o\left(x^{-1} \ln \left(\frac{x}{T_{*}^{2}}\right)\right)+O\left(T_{*}^{4} x^{-2}\right)$. Now choosing $T_{*}<x^{1 / 4}$, such that $o\left(x^{-1} \ln \left(\frac{x}{T_{*}^{2}}\right)\right)=o\left(x^{-1}\right)$, substituting (3.14), (3.15) and (3.17) into (3.13), and combining the latter with (3.2), we obtain (3.11), thus completing the proof of Lemma 2 .

For large $x$, it follows from Theorem 2 that there are two uniform asymptotic solutions of equation (2.9) $\tilde{\phi}_{\nu}$ and $\tilde{\phi}_{-\nu-1}$, namely

$$
\tilde{\phi}_{\nu}(\lambda, x)=\left(\frac{\zeta^{2}-\alpha^{2}}{Q(\lambda, x)}\right)^{\frac{1}{4}} D_{\nu}\left(e^{\pi i / 4} \sqrt{2 x} \zeta\right)
$$


and

$$
\tilde{\phi}_{-\nu-1}(\lambda, x)=\left(\frac{\zeta^{2}-\alpha^{2}}{Q(\lambda, x)}\right)^{\frac{1}{4}} D_{-\nu-1}\left(e^{-\pi i / 4} \sqrt{2 x} \zeta\right),
$$

the uniformity is with respect to $\lambda$ on the Stokes curves.

We denote by $\hat{\Psi}_{i j}$ the $(i, j)$ entry of $\hat{\Psi}$ and seek asymptotic solutions at infinity and at the origin. By virtue of (2.5), we have

$$
\begin{aligned}
& \hat{\Psi}_{11}^{(\infty)}=B^{1 / 2}\left(c_{1} \tilde{\phi}_{\nu}+c_{2} \tilde{\phi}_{-\nu-1}\right), \\
& \hat{\Psi}_{12}^{(\infty)}=B^{1 / 2}\left(c_{3} \tilde{\phi}_{\nu}+c_{4} \tilde{\phi}_{-\nu-1}\right),
\end{aligned}
$$

where $c_{j}, j=1,2,3,4$ are constants to be determined by (1.18).

Similarly, we obtain

$$
\begin{aligned}
& \hat{\Psi}_{11}^{(0)}=B^{1 / 2}\left(c_{5} \tilde{\phi}_{\nu}+c_{6} \tilde{\phi}_{-\nu-1}\right), \\
& \hat{\Psi}_{12}^{(0)}=B^{1 / 2}\left(c_{7} \tilde{\phi}_{\nu}+c_{8} \tilde{\phi}_{-\nu-1}\right),
\end{aligned}
$$

where again $c_{j}, j=5,6,7,8$ are constants to be determined.

For our purpose, we need to determine the asymptotic values of $c_{j}, j=1, \cdots, 8$. As a matter of fact, it follows from the first equality in (1.20) that

$$
\begin{aligned}
& \hat{\Psi}_{11}^{(\infty)}=\left(E_{0}\right)_{11} \hat{\Psi}_{11}^{(0)}+\left(E_{0}\right)_{21} \hat{\Psi}_{12}^{(0)}, \\
& \hat{\Psi}_{12}^{(\infty)}=\left(E_{0}\right)_{12} \hat{\Psi}_{11}^{(0)}+\left(E_{0}\right)_{22} \hat{\Psi}_{12}^{(0)} .
\end{aligned}
$$

We obtain from (3.20), (3.22)-(3.23) and (3.24) that

$$
c_{1}=\left(E_{0}\right)_{11} c_{5}+\left(E_{0}\right)_{21} c_{7}, c_{2}=\left(E_{0}\right)_{11} c_{6}+\left(E_{0}\right)_{21} c_{8},
$$

which in turn gives

$$
\frac{\left(E_{0}\right)_{11}}{\left(E_{0}\right)_{21}}=-\frac{c_{1} c_{8}-c_{2} c_{7}}{c_{1} c_{6}-c_{2} c_{5}}
$$

Similarly calculation leads to

$$
\frac{\left(E_{0}\right)_{22}}{\left(E_{0}\right)_{12}}=-\frac{c_{3} c_{6}-c_{4} c_{5}}{c_{3} c_{8}-c_{4} c_{7}}
$$

Eventually, substituting (3.26) and (3.27) into (1.24) yields

$$
I_{0}=\frac{\left(c_{1} c_{8}-c_{2} c_{7}\right)\left(c_{3} c_{6}-c_{4} c_{5}\right)}{\left(c_{3} c_{8}-c_{4} c_{7}\right)\left(c_{1} c_{6}-c_{2} c_{5}\right)} .
$$

Now, we are in a position to calculate $c_{j}$ for $j=1, \cdots, 8$.

From [21, Sec.12.9], we have the asymptotic behavior of $D_{\nu}(z)$ for $|z| \rightarrow \infty$ as follows:

$$
D_{\nu}(z) \sim \begin{cases}z^{\nu} e^{-\frac{1}{4} z^{2}}, & \arg z \in\left(-\frac{3}{4} \pi, \frac{3}{4} \pi\right), \\ z^{\nu} e^{-\frac{1}{4} z^{2}}-\frac{\sqrt{2 \pi}}{\Gamma(-\nu)} e^{i \pi \nu} z^{-\nu-1} e^{\frac{1}{4} z^{2}}, & \arg z \in\left(\frac{1}{4} \pi, \frac{5}{4} \pi\right) .\end{cases}
$$


and the asymptotic behavior of $D_{-\nu-1}(i z)$ for $|z| \rightarrow \infty$ is that

$$
D_{-\nu-1}(i z) \sim \begin{cases}e^{-\pi i(\nu+1) / 2} z^{-\nu-1} e^{\frac{1}{4} z^{2}}, & \text { on } \arg z=-\frac{1}{4} \pi, \\ -\frac{\sqrt{2 \pi}}{\Gamma(\nu+1)} e^{-\pi i(\nu+2) / 2} z^{\nu} e^{-\frac{1}{4} z^{2}}, & \text { on } \arg z=\frac{1}{4} \pi .\end{cases}
$$

For $\lambda$ on the Stokes line $\arg \lambda=-\frac{\pi}{2}$ and $|\lambda| \rightarrow \infty$, it immediately follows from (3.1) that $\zeta^{2} \sim i \lambda$, if we take $i=e^{\frac{\pi i}{2}}$, then we have $\arg \zeta \sim 0$. Therefore, $\arg \left(e^{\pi i / 4} \sqrt{2 x} \zeta\right) \sim \frac{\pi}{4}$ and $\arg \left(e^{-\pi i / 4} \sqrt{2 x} \zeta\right) \sim-\frac{\pi}{4}$ for $x>0$. Taking $-1=e^{\pi i}$, from (2.9) we have $Q^{-1 / 4} \sim$ $\sqrt{2} e^{-\pi i / 4}$ as $|\lambda| \rightarrow \infty$. Since $\left(\zeta^{2}-\alpha^{2}\right)^{1 / 4} \sim \zeta^{1 / 2}$ as $|\lambda| \rightarrow \infty$, using the appropriate asymptotic formulas of $D_{\nu}(z)$ in (3.29), and in view of (3.18) and (3.1), we have

$$
\tilde{\phi}_{\nu} \sim A_{0} e^{\frac{x}{2} \lambda}, \text { as }|\lambda| \rightarrow \infty .
$$

Here use has been made of $s=\frac{x}{4}-a b \ln \frac{x}{4}$, and

$$
A_{0}=\sqrt{2} e^{-\pi i / 4} x^{-1 / 4} e^{-\frac{1}{4} x} 2^{3 i a b-1 / 2} b^{-1 / 2} e^{-\pi a b} e^{\frac{\pi i}{4} \nu} .
$$

Similarly, by applying the appropriate asymptotic formulas of $D_{\nu}(z)$ in (3.29), we obtain from (3.19) and (3.1) that

$$
\tilde{\phi}_{-\nu-1} \sim B_{0} e^{-\frac{x}{2} \lambda} \text {, as }|\lambda| \rightarrow \infty,
$$

where

$$
B_{0}=\sqrt{2} e^{-\pi i / 4} x^{-1 / 4} e^{\frac{1}{4} x} 2^{-3 i a b} b^{1 / 2} e^{\pi a b} e^{\frac{\pi i}{4}(\nu+1)} .
$$

On the other hand, by virtue of (2.1), we obtain from (1.18) that

$$
\begin{aligned}
& \hat{\Psi}_{11}^{(\infty)} \sim \frac{1}{\sqrt{2}} u^{-\frac{1}{2}} e^{\frac{x}{2} \lambda}, \\
& \hat{\Psi}_{12}^{(\infty)} \sim \frac{1}{\sqrt{2}} i u^{\frac{1}{2}} e^{-\frac{x}{2} \lambda},
\end{aligned}
$$

Thus, applying (3.31) and (3.32), a combination of (3.20), (3.21), (3.33) and (3.34) implies

$$
c_{2}=c_{3}=0
$$

Substituting (3.35) into (3.28) gives the simplified version

$$
I_{0}=\frac{c_{5} c_{8}}{c_{6} c_{7}}
$$

Our next task is to determine $c_{j}$ for $j=5,6,7,8$.

By using the result from [2, Prop.7.1], we have

$$
\hat{\Psi}^{(0)} \sim M_{1}\left(\begin{array}{cc}
v+\Theta & v+\Theta \\
v & v+\Theta
\end{array}\right)(I+o(1)) \Phi\left(\frac{1}{2} x \sqrt{\lambda}, \Theta\right) u^{-\frac{1}{2} \sigma_{3}},
$$


as $\lambda \rightarrow 0$ and $x \rightarrow \infty$, where $M_{1}$ is defined in (2.3) and

$$
\Phi(x, \Theta)=x\left(\begin{array}{cc}
J_{\Theta-1}(x) & J_{1-\Theta}(x) \\
J_{\Theta+1}(x) & J_{-(\Theta+1)}(x)
\end{array}\right)
$$

where $J_{\mu}(x)$ is the Bessel function of the first kind, and $\Theta \notin \mathbb{Z}$, as mentioned earlier.

For $\arg \lambda=2 \pi$, choosing $\lambda \rightarrow 0$ such that $x \sqrt{\lambda} \rightarrow \infty$ and $x \zeta^{2} \rightarrow \infty$, by virtue of the asymptotic expansion of $v(x)$ in (2.7) and the behavior for Bessel function

$$
J_{\mu}(z)=\sqrt{\frac{2}{\pi z}}\left[\cos \left(z-\frac{1}{2} \mu \pi-\frac{1}{4} \pi\right)-\frac{1}{2 z}\left(\mu^{2}-\frac{1}{4}\right) \sin \left(z-\frac{1}{2} \mu \pi-\frac{1}{4} \pi\right)+O\left(z^{-2}\right)\right],
$$

as $z \rightarrow \infty$ for $|\arg z|<\pi$; cf. [21, 26], a straightforward calculation enables us to write (3.37) as the following form

$\hat{\Psi}^{(0)} \sim \frac{-\Theta}{2 \sqrt{\pi}} x^{1 / 2} \lambda^{-1 / 4} M_{1}\left(\begin{array}{cc}\cos \left(\frac{1}{2} x \sqrt{\lambda}-\frac{1}{2} \Theta \pi-\frac{1}{4} \pi\right) & -\cos \left(\frac{1}{2} x \sqrt{\lambda}+\frac{1}{2} \Theta \pi-\frac{1}{4} \pi\right) \\ \cos \left(\frac{1}{2} x \sqrt{\lambda}-\frac{1}{2} \Theta \pi-\frac{1}{4} \pi\right) & -\cos \left(\frac{1}{2} x \sqrt{\lambda}+\frac{1}{2} \Theta \pi-\frac{1}{4} \pi\right)\end{array}\right) u^{-\frac{1}{2} \sigma_{3}}$,

which gives us

$$
\hat{\Psi}_{11}^{(0)} \sim-\frac{\Theta}{4 \sqrt{\pi}} x^{1 / 2} u^{-\frac{1}{2}} \lambda^{-1 / 4}(1+i)\left(e^{-\frac{i}{2} \Theta \pi-\frac{i}{4} \pi} e^{\frac{i}{2} x \sqrt{\lambda}}+e^{\frac{i}{2} \Theta \pi+\frac{i}{4} \pi} e^{-\frac{i}{2} x \sqrt{\lambda}}\right)
$$

and

$$
\hat{\Psi}_{12}^{(0)} \sim \frac{\Theta}{4 \sqrt{\pi}} x^{1 / 2} u^{\frac{1}{2}} \lambda^{-1 / 4}(1+i)\left(e^{\frac{i}{2} \Theta \pi-\frac{i}{4} \pi} e^{\frac{i}{2} x \sqrt{\lambda}}+e^{-\frac{i}{2} \Theta \pi+\frac{i}{4} \pi} e^{-\frac{i}{2} x \sqrt{\lambda}}\right)
$$

as $x \sqrt{\lambda} \rightarrow \infty$.

On the other hand, for $\arg \lambda=2 \pi$, choosing $\lambda \rightarrow 0$ such that $x \sqrt{\lambda} \rightarrow \infty$ and $x \zeta^{2} \rightarrow \infty$, then we have from (3.11) that $\zeta^{2} \sim \sqrt{\lambda}$, which gives us $\arg \zeta \sim \frac{1}{2} \pi$. Hence, $\arg \left(e^{\pi i / 4} \sqrt{2 x} \zeta\right) \sim \frac{3 \pi}{4}$ and $\arg \left(e^{-\pi i / 4} \sqrt{2 x} \zeta\right) \sim \frac{\pi}{4}$ for $x>0$. From (2.9) we have $Q^{-1 / 4} \sim$ $2 \lambda^{1 / 4}$ as $|\lambda| \rightarrow 0$. By using the appropriate asymptotic formulas of $D_{\nu}(z)$ in (3.29), we obtain from (3.18) and (3.11) that

$$
\tilde{\phi}_{\nu} \sim 2 \lambda^{1 / 4} x^{-1 / 4}\left(C_{0} e^{-\frac{i}{2} x \sqrt{\lambda}}-D_{0} e^{\frac{i}{2} x \sqrt{\lambda}}\right),
$$

where

$$
C_{0}=e^{\pi i(\nu+1) / 4} 2^{3 i a b-1 / 2} b^{-1 / 2}, \quad D_{0}=\frac{\sqrt{2 \pi}}{\Gamma(-\nu)} e^{\frac{3 i \pi}{4} \nu} e^{-\frac{\pi i}{2}} 2^{-3 i a b} b^{1 / 2}
$$

Similarly, by further applying the appropriate asymptotic formulas for $D_{-\nu-1}(-i z)$ in (3.30), we obtain from (3.19) and (3.11) that

$$
\tilde{\phi}_{-\nu-1} \sim 2 \lambda^{1 / 4} x^{-1 / 4} F_{0} e^{\frac{i}{2} x \sqrt{\lambda}}
$$


with

$$
F_{0}=e^{\frac{\pi i}{4} \nu} 2^{-3 i a b} b^{1 / 2}
$$

Substituting (3.41) and (3.43) into (3.22) and using (3.39), we obtain

$$
\begin{aligned}
& B^{1 / 2} 2 \lambda^{1 / 4} x^{-1 / 4} c_{5} \sim-\frac{\Theta}{4 \sqrt{\pi}} x^{1 / 2} u^{-\frac{1}{2}} \lambda^{-1 / 4}(1+i) e^{\frac{i}{2} \Theta \pi+\frac{i}{4} \pi} C_{0}^{-1} \\
& B^{1 / 2} 2 \lambda^{1 / 4} x^{-1 / 4} c_{6} F_{0} \sim-\frac{\Theta}{4 \sqrt{\pi}} x^{1 / 2} u^{-\frac{1}{2}} \lambda^{-1 / 4}(1+i)\left(e^{-\frac{i}{2} \Theta \pi-\frac{i}{4} \pi}+e^{\frac{i}{2} \Theta \pi+\frac{i}{4} \pi} D_{0} C_{0}^{-1}\right) .
\end{aligned}
$$

Similarly, substituting (3.41) and (3.43) into (3.23) and using (3.40), we obtain

$$
\begin{aligned}
& B^{1 / 2} 2 \lambda^{1 / 4} x^{-1 / 4} c_{7} \sim \frac{\Theta}{4 \sqrt{\pi}} x^{1 / 2} u^{\frac{1}{2}} \lambda^{-1 / 4}(1+i) e^{-\frac{i}{2} \Theta \pi+\frac{i}{4} \pi} C_{0}^{-1} \\
& B^{1 / 2} 2 \lambda^{1 / 4} x^{-1 / 4} c_{8} F_{0} \sim \frac{\Theta}{4 \sqrt{\pi}} x^{1 / 2} u^{\frac{1}{2}} \lambda^{-1 / 4}(1+i)\left(e^{\frac{i}{2} \Theta \pi-\frac{i}{4} \pi}+e^{-\frac{i}{2} \Theta \pi+\frac{i}{4} \pi} D_{0} C_{0}^{-1}\right) .
\end{aligned}
$$

Therefore, taking ratios from (3.45) and (3.47), we have

$$
\frac{c_{5}}{c_{7}}=-e^{\pi i \Theta} u^{-1}
$$

and a parallel result follows from (3.46) and (3.48) reads

$$
\frac{c_{8}}{c_{6}}=-u \frac{e^{\frac{i}{2} \Theta \pi-\frac{i}{4} \pi}+e^{-\frac{i}{2} \Theta \pi+\frac{i}{4} \pi} D_{0} C_{0}^{-1}}{e^{-\frac{i}{2} \Theta \pi-\frac{i}{4} \pi}+e^{\frac{i}{2} \Theta \pi+\frac{i}{4} \pi} D_{0} C_{0}^{-1}},
$$

where

$$
D_{0} C_{0}^{-1}=-\frac{2 \sqrt{\pi} b}{\Gamma(1-2 i a b)} e^{-\frac{i}{4} \pi} e^{-\pi a b} 2^{-6 i a b} .
$$

Substituting (3.49) and (3.50) into (3.36), we obtain the first invariant quantity for large $x$

$$
I_{0}=\frac{i e^{\pi i \Theta}-D_{0} C_{0}^{-1}}{i e^{-\pi i \Theta}-D_{0} C_{0}^{-1}} .
$$

For large $x$, in the same manner, the other invariant can also be calculated as

$$
I_{1}=\frac{i e^{\pi i \Theta}-\frac{2 \sqrt{\pi} a}{\Gamma(1+2 i a b)} e^{\frac{i}{4} \pi} e^{-\pi a b} 2^{6 i a b}}{i e^{-\pi i \Theta}-\frac{2 \sqrt{\pi} a}{\Gamma(1+2 i a b)} e^{\frac{i}{4} \pi} e^{-\pi a b} 2^{6 i a b}} .
$$

A combination of (1.26) and (3.52) with (3.51) gives (1.10), and a combination of (1.27) and (3.53) gives (1.9). 


\section{Acknowledgements}

Yu-Qiu Zhao was supported in part by the National Natural Science Foundation of China under grant number 10871212 .

\section{References}

[1] F.V. Andreev, A.V. Kitaev, On connection formulas for the asymptotics of some special solutions of the fifth Painlevé equation, Zap. Nauchn. Sem. POMI 243 (1997) 19-29.

[2] F.V. Andreev, A.V. Kitaev, Connection formulae for asymptotics of the fifth Painlevé transcendent on the real axis, Nonlinearity 13 (2000) 1801-1840 .

[3] A. Bobenko, U. Eitner, A. Kitaev, Surfaces with harmonic inverse mean curvature and Painlevé equations, Geom. Dedicata 68 (1997) 187-227.

[4] A.P. Bassom, P.A. Clarkson, C.K. Law and J.B. McLeod, Application of uniform asymptotics to the second Painlevé transcendent, Arch. Rational Mech. Anal. 143 (1998) 241-271.

[5] D.B. Creamer, H.B. Thacker and D. Wilkinson, Some exact results for the two-point function of an integrable quantum field theory, Phys. Rev. D 23 (1981) 3081-3084.

[6] T.M. Dunster, Asymptotic solutions of second-order linear differential equations having almost coalescent turning points, with an application to the incomplete gamma function, Proc. Roy. Soc. London Ser. A 452 (1996) 1331-1349.

[7] A.S. Fokas, A.R. Its, A.A. Kapaev and V.Yu. Novokshenov, Painlevé transcendents. The Riemann-Hilbert approach, Mathematical Surveys and Monographs, Vol. 128, Amer. Math. Soc., Providence RI, 2006.

[8] H. Flaschka and A.C. Newell, Monodromy- and spectrum-preserving deformations. I, Comm. Math. Phys. 76 (1980) 65-116.

[9] A.R. Its and V.Yu. Novokshenov, The isomonodromic deformation method in the theory of Painlevé equations: Lecture Notes in Mathematics 1191 Springer, Berlin, 1986.

[10] M. Jimbo, Monodromy problem and the boundary condition for some Painlevé equations, Publ. Res. Inst. Math. Sci. 18 (1982) 1137-1161.

[11] G.L. Lamb Jr.,Elements of soliton theory, John Wiley and Sons, New York, 1980. 
[12] Y.-M. Lu and Z.-D. Shao, Application of uniform asymptotics method to the asymptotics of the solutions of the fifth Painlevé equation when $\delta=0$, Appl. Anal. 79 (2001) 335-350.

[13] Y.-M. Lu and Z.-D. Shao, Application of uniform asymptotics to the fifth Painlevé transcendant, Int. J. Math. Math. Sci. 31 (2002) 43-49.

[14] B.M. McCoy, J.H.H. Perk and R.E. Shrock, Time-dependent correlation functions of the transverse Ising chain at the critical magnetic field, Nuclear Physics B $\mathbf{2 2 0}$ (1983) 35-47.

[15] B.M. McCoy, J.H.H. Perk and R.E. Shrock, Correlation functions of the transverse Ising chain at the critical field for large temporal and spatial separations, Nuclear Physics B 220 (1983) 269-282.

[16] B.M. McCoy, C.A. Tracy and T.T. Wu, Painlevé functions of the third kind, J. Math. Phys. 18 (1977) 1058-1092.

[17] B.M. McCoy and S. Tang, Connection formulae for Painlevé V functions, Phys. D 19 (1986) 42-72.

[18] B.M. McCoy and S. Tang, Connection formulae for Painlevé V functions. II. the $\delta$ function Bose gas problem, Phys. D 20 (1986) 187-216.

[19] B.M. McCoy and S. Tang, Connection formulae for Painlevé functions, Phys. D 18 (1986) 190-196.

[20] F.W.J. Olver, Second-order linear differential equations with two turning points, Philos. Trans. Roy. Soc. London Ser. A 278 (1975) 137-174.

[21] F.W.J. Olver, D.W. Lozier, R.F. Boisvert and C.W. Clark (eds.), NIST Handbook of Mathematical Functions, Cambridge University Press, Cambridge, UK, 2010.

[22] H.-Z. Qin and Y.-M. Lu, Application of uniform asymptotics method to analyzing the asymptotic behaviour of the general fourth Painlevé transcendent, Int. J. Math. Math. Sci. 2005 (2005) 1421-1434.

[23] B.I. Suleimanov, On asymptotics of regular solutions for a special kind of Painlevé V equation, Lecture Notes in Mathematics 1193 230-260, 1986.

[24] C. Tracy and H. Widom, Level spacing distributions and the Bessel kernel, Comm. Math. Phys., 161 (1994) 289-309.

[25] H.G. Vaidya and C.A. Tracy, One particle reduced density matrix of impenetrable bosons in one dimension at zero temperature, J. Math. Phys. 20 (1979) 2291-2312. 
[26] E.T. Whittaker and G.M. Watson, A course of modern analysis, 4th ed., Cambridge University Press, Cambridge, 1927.

[27] R. Wong and H.-Y. Zhang, On the connection formulas of the third Painlevé transcendent, Discrete Contin. Dyn. Syst. 23 (2009) 541-560.

[28] R. Wong and H.-Y. Zhang, On the connection formulas of the fourth Painlevé transcendent, Anal. Appl. 7 (2009) 419-448. 